\title{
ALGEBRAIC AND TRIANGULAR $n$-HYPONORMAL OPERATORS
}

\author{
EUNGIL KO
}

(Communicated by Palle E. T. Jorgensen)

\begin{abstract}
In this paper we shall prove that if an operator $T \in \mathscr{L}\left(\bigoplus_{1}^{n} \mathbf{H}\right)$ is a finite triangular operator matrix with hyponormal operators on main diagonal, then $T$ is subscalar. As corollaries we get the following:

(1) Every algebraic operator is subscalar.

(2) Every operator on a finite-dimensional complex space is subscalar.

(3) Every triangular $n$-hyponormal operator is subscalar.
\end{abstract}

\section{INTRODUCTION}

Let $\mathbf{H}$ and $\mathbf{K}$ be separable, complex Hilbert spaces and $\mathscr{L}(\mathbf{H}, \mathbf{K})$ denote the space of all linear, bounded operators from $\mathbf{H}$ to $\mathbf{K}$. If $\mathbf{H}=\mathbf{K}$, we write $\mathscr{L}(\mathbf{H})$ in place of $\mathscr{L}(\mathbf{H}, \mathbf{K})$. An operator $T$ in $\mathscr{L}(\mathbf{H})$ is called hyponormal if $T T^{*} \leq T^{*} T$ or, equivalently, if $\left\|T^{*} h\right\| \leq\|T h\|$ for each $h$ in $\mathbf{H}$.

A linear bounded operator $S$ on $\mathbf{H}$ is called scalar of order $m$ if it possesses a spectral distribution of order $m$, i.e., if there is a continuous unital morphism of topological algebras

$$
\Phi: C_{0}^{m}(\mathbf{C}) \rightarrow \mathscr{L}(\mathbf{H})
$$

such that $\Phi(z)=S$, where as usual $z$ stands for the identity function on $\mathbf{C}$ and $C_{0}^{m}(\mathbf{C})$ stands for the space of compactly supported functions on $\mathbf{C}$, continuously differentiable of order $m, 0 \leq m \leq \infty$. An operator is subscalar if it is similar to the restriction of a scalar operator to a closed invariant subspace.

This paper is divided into four sections. Section 2 deals with some preliminary facts. In section 3, we shall state the Putinar theorem. In section 4, we shall prove our main theorem and several corollaries.

\section{Preliminaries}

An operator $T \in \mathscr{L}(\mathbf{H})$ is said to satisfy the single-valued extension property if for any open subset $U$ in $\mathbf{C}$, the function

$$
z-T: O(U, \mathbf{H}) \rightarrow O(U, \mathbf{H})
$$

defined by the obvious pointwise multiplication is one-to-one where $O(U, \mathbf{H})$ denotes the Fréchet space of $\mathbf{H}$-valued analytic functions on $U$ with respect to

Received by the editors March 2, 1994 and, in revised form, June 13, 1994; the results of this paper were delivered in a talk at the Korean Mathematical Society Spring Conference in April 1994.

1991 Mathematics Subject Classification. Primary 47B20; Secondary 47B38.

The author was partially supported by GARC. 
uniform topology. If, in addition, the above function $z-T$ has closed range on $O(U, \mathbf{H})$, then $T$ satisfies the Biship's condition $(\beta)$.

In other terms, condition $(\beta)$ means that, for any open set $U$ and any sequence of analytic functions $f_{n} \in O(U, \mathbf{H}), \lim _{n \rightarrow \infty} f_{n}=0$ in $O(U, \mathbf{H})$ whenever $\lim _{n \rightarrow \infty}(z-T) f_{n}=0$. In particular, $(z-T) g=0$ if and only if $g=0$, where $g \in O(U, \mathbf{H})$.

2.1 Lemma ([MP], Theorem 5.5). Every hyponormal operator has property $(\beta)$.

Let $z$ be the coordinate in the complex plane $\mathbf{C}$, and let $d \mu(z)$, or simply $d \mu$, denote the planar Lebesgue measure. Fix a complex (separable) Hilbert space $\mathbf{H}$ and a bounded (connected) open subset $U$ of $\mathbf{C}$. We shall denote by $L^{2}(U, \mathbf{H})$ the Hilbert space of measurable functions $f: U \rightarrow \mathbf{H}$, such that

$$
\|f\|_{2, U}=\left(\int_{U}\|f(z)\|^{2} d \mu(z)\right)^{1 / 2}<\infty .
$$

The space of functions $f \in L^{2}(U, \mathbf{H})$ which are analytic functions in $U$ (i.e., $\bar{\partial} f=0$ ) is denoted by

$$
A^{2}(U, \mathbf{H})=L^{2}(U, \mathbf{H}) \cap O(U, \mathbf{H}) .
$$

$A^{2}(U, \mathbf{H})$ is called the Bergman space for $U$. Note that $A^{2}(U, \mathbf{H})$ is complete (i.e., $A^{2}(U, \mathbf{H})$ is a Hilbert space).

Let $P$ denote the orthogonal projection of $L^{2}(U, \mathbf{H})$ onto $A^{2}(U, \mathbf{H})$. Let $L^{\infty}(U, \mathbf{H})$ denote the Banach space of essentially bounded $\mathbf{H}$-valued functions on $U$. Let $\bar{U}$ be the closure in $\mathbf{C}$ of the open set $U$, and let $C^{p}(\bar{U}, \mathbf{H})$ denote the space of continuously differentiable functions on $\bar{U}$ of order $p, 0 \leq p \leq \infty$.

Cauchy-Pompeiu formula. For a bounded disk $D$,

$$
f(z)=\frac{1}{2 \pi i} \int_{\partial D} \frac{f(s)}{s-z} d s-\frac{1}{\pi} \int_{D} \frac{\bar{\partial} f(s)}{s-z} d \mu(s)
$$

where $z \in D$ and $f \in C^{2}(\bar{D}, \mathbf{H})$.

Remark. The function $g(z)=\int_{\partial D} \frac{f(s)}{s-z} d \mu(s)$ appearing in the Cauchy-Pompeiu formula is analytic in $D$ and continuous on $\bar{D}$, in particular $g \in A^{2}(D, \mathbf{H})$ for $f \in C^{2}(\bar{D}, \mathbf{H})$.

Let us now define a special Sobolev type space. Let $U$ again be a bounded open subset of $\mathbf{C}$ and $m$ be a fixed non-negative integer. The vector-valued Sobolev space $W^{m}(U, \mathbf{H})$ with respect to $\bar{\partial}$ and of order $m$ will be the space of those functions $f \in L^{2}(U, \mathbf{H})$ whose derivatives $\bar{\partial} f, \ldots, \bar{\partial}^{n} f$ in the sense of distributions still belong to $L^{2}(U, \mathbf{H})$. Endowed with the norm

$$
\|f\|_{W^{m}}^{2}=\sum_{i=0}^{m}\left\|\bar{\partial}^{i} f\right\|_{2, U}^{2}
$$

$W^{m}(U, \mathbf{H})$ becomes a Hilbert space contained continuously in $L^{2}(U, \mathbf{H})$.

Let $U$ be a (connected) bounded open subset of $\mathbf{C}$, and let $m$ be a nonnegative integer. The linear operator $M$ of multiplication by $z$ on $W^{m}(U, \mathbf{H})$ is continuous and has a spectral distribution of order $m$, defined by the relation

$$
\Phi_{M}: C_{0}^{m}(\mathbf{C}) \rightarrow \mathscr{L}\left(W^{m}(U, \mathbf{H})\right), \quad \Phi_{M}(f)=M_{f} .
$$


Therefore, $M$ is a scalar operator of order $m$. Let

$$
V: W^{m}(U, \mathbf{H}) \rightarrow \bigoplus_{0}^{m} L^{2}(U, \mathbf{H})
$$

be the operator $V(f)=\left(f, \bar{\partial} f, \ldots, \bar{\partial}^{m} f\right)$. Then $V$ is an isometry such that $V M_{z}=\left(\bigoplus_{0}^{m} M_{z}\right) V$. Therefore, $M_{z}$ is a subnormal operator.

\section{Putinar's theorem}

Let $T$ be in $\mathscr{L}(\mathbf{H})$. Then for a given open bounded subset $D$ of $\mathbf{C}, z-T$ acts (linearly and) continuously on the space $W^{2}(D, \mathbf{H})$.

3.1 Lemma ([Pu], Lemma 1.1). If $U$ and $V$ are bounded connected open sets in $\mathbf{C}$, and if $V$ is relatively compact in $U$, then there is a constant $c>0$, such that

$$
\|f\|_{\infty, V} \leq c\|f\|_{2, U}
$$

for every $f$ in $A^{2}(U, \mathbf{H})$.

3.2 Proposition ([Pu], Proposition 2.1). For a bounded disk $D$ in complex plane there is a constant $C_{D}$, such that for an arbitrary operator $T$ in $\mathscr{L}(\mathbf{H})$ and $f$ in $W^{2}(D, \mathbf{H})$ we have

$$
\|(I-P) f\|_{2, D} \leq C_{D}\left(\left\|(z-t)^{*} \bar{\partial} f\right\|_{2, D}+\left\|(z-T)^{*} \bar{\partial}^{2} f\right\|_{2, D}\right)
$$

where $P$ denotes the orthogonal projection of $L^{2}(D, \mathbf{H})$ onto the Bergman space $A^{2}(D, \mathbf{H})$.

3.3 Corollary ([Pu], Corollary 2.2). If $T$ is hyponormal, then

$$
\|(I-P) f\|_{2, D} \leq C_{D}\left(\|(z-T) \bar{\partial} f\|_{2, D}+\left\|(z-T) \bar{\partial}^{2} f\right\|_{2, D}\right) .
$$

3.4 Theorem ([Pu], Theorem 1). Any hyponormal operator is subscalar of order 2.

Proof. Let $T$ be a hyponormal operator on the Hilbert space $\mathbf{H}$. Consider an arbitrary bounded open subset $D$ of $\mathbf{C}$ and the quotient space

$$
H(D)=\frac{W^{2}(D, \mathbf{H})}{\operatorname{cl}(z-T) W^{2}(D, \mathbf{H})}
$$

endowed with the Hilbert space norm. The class of a vector $f$ or an operator $A$ on this quotient will be denoted by $\tilde{f}$, respectively $\tilde{A}$.

Note that $M$, the operator of multiplication by $z$ on $W^{2}(D, \mathbf{H})$, leaves invariant $\operatorname{ran}(z-T)$, hence $\widetilde{M}$ is well defined.

On the other hand, the map

$$
\Phi: C_{0}^{2}(\mathbf{C}) \rightarrow \mathscr{L}\left(W^{2}(D, \mathbf{H})\right), \quad \boldsymbol{\Phi}(f)=M_{f}
$$

is a spectral distribution for $M$, of order 2. Thus the operator $M$ is $C^{2}$-scalar. Since $\overline{\operatorname{ran}(z-T)}$ is invariant under every operator $M_{f}, f \in C_{0}^{2}(\mathbf{C})$, we infer that $\widetilde{M}$ is a $C^{2}$-scalar operator with spectral distribution $\Phi$.

Define

$$
V: \mathbf{H} \rightarrow \frac{W^{2}(D, \mathbf{H})}{\operatorname{cl}(z-T) W^{2}(D, \mathbf{H})}
$$


by $V(h)=1 \tilde{\otimes} h$ where $1 \otimes h$ denotes the constant $h$. Then

$$
V T=\widetilde{M} V .
$$

Indeed, $V T h=(1 \otimes T h)^{\sim}=(z \otimes h)^{\sim}=\widetilde{M}(1 \otimes h)^{\sim}=\widetilde{M} V h$. In particular $\operatorname{ran} V$ is an invariant subspace for $\widetilde{M}$. In order to conclude the proof of this theorem, it is enough to show the following lemma.

3.5 Lemma ([Pu], Lemma 2.3). Let $D$ be a bounded disk which contains $\sigma(T)$. Then the operator $V$ is one-to-one and has closed range.

Proof. We have to prove the following assertion: If $h_{n}$ in $\mathbf{H}$ and $f_{n}$ in $W^{2}(D, \mathbf{H})$ are sequences such that

$$
\lim _{n \rightarrow \infty}\left\|(z-T) f_{n}+h_{n}\right\|_{W^{2}}=0,
$$

then $\lim _{n \rightarrow \infty} h_{n}=0$. The assumption (1) implies

$$
\lim _{n \rightarrow \infty}\left(\left\|(z-T) \bar{\partial} f_{n}\right\|_{2, D}+\left\|(z-T) \bar{\partial}^{2} f_{n}\right\|_{2, D}\right)=0 .
$$

By Corollary 3.3,

$$
\lim _{n \rightarrow \infty}\left\|(I-P) f_{n}\right\|_{2, D}=0
$$

Then by (1),

$$
\lim _{n \rightarrow \infty}\left\|(z-T) P f_{n}+h_{n}\right\|_{2, D}=0 .
$$

Let $\Gamma$ be a curve in $D$ surrounding $\sigma(T)$. Then for $z \in \Gamma$

$$
\lim _{n \rightarrow \infty}\left\|P f_{n}(z)+(z-T)^{-1} h_{n}\right\|=0
$$

uniformly by the preceding consequence of Proposition 3.2. Hence,

$$
\left\|\frac{1}{2 \pi i} \int_{\Gamma} P f_{n}(z) d z+h_{n}\right\| \rightarrow 0 .
$$

But $\int_{\Gamma} P f_{n} d z=0$. Hence, $\lim _{n \rightarrow \infty} h_{n}=0$.

\section{MAIN THEOREMS}

In this section, we shall prove that every algebraic and triangular $n$-hyponormal operator is subscalar.

4.1 Definition. An operator $T \in \mathscr{L}(\mathbf{H})$ is algebraic if there is a non-zero polynomial $p$ such that $p(T)=0$.

4.2 Definition. An operator $T \in \mathscr{L}(\mathbf{H})$ is nilpotent if $T^{n}=0$ for some integer $n$.

4.3 Proposition. Every nilpotent operator is an algebraic operator.

An interesting characterization of algebraic operators was given by P. R. Halmos. 
4.4 Theorem ([Ha]). If $T$ is an algebraic operator and $p$ is a polynomial of minimal degree $n$ such that $p(T)=0$, then $T$ is unitarily equivalent to a finite operator matrix of the form

$$
\left(\begin{array}{ccccc}
\alpha_{1} & T_{12} & \ldots & \ldots & T_{1 n} \\
0 & \alpha_{2} & T_{23} & \ldots & \ldots \\
\ldots & \ldots & \ldots & \ldots & \\
\ldots & \ldots & \ldots & \alpha_{n-1} & T_{n-1, n} \\
0 & \ldots & \ldots & 0 & \alpha_{n}
\end{array}\right)
$$

where $\alpha_{i}$ are the roots of the polynomial $p$.

The following theorem will be proved in this paper.

4.5 Theorem. If an operator $T \in \mathscr{L}\left(\bigoplus_{1}^{n} \mathbf{H}\right)$ is a finite operator matrix of the form

$$
T=\left(\begin{array}{ccccc}
T_{11} & T_{12} & \ldots & \ldots & T_{1 n} \\
0 & T_{22} & T_{23} & \ldots & \ldots \\
\ldots & \ldots & \ldots & \ldots & \ldots \\
\ldots & \ldots & \ldots & T_{n-1, n-1} & T_{n-1, n} \\
0 & \ldots & \ldots & 0 & T_{n, n}
\end{array}\right)
$$

where $T_{i, i}$ are hyponormal for $i=1,2, \ldots, n$, then $T$ is a subscalar operator of order $2 n$.

Proof. Consider an arbitrary bounded open subset $D$ of $\mathbf{C}$ which contains $\sigma(T)$ and the quotient space

$$
H(D)=\frac{\oplus_{1}^{n} W^{2 n}(D, \mathbf{H})}{\operatorname{cl}(T-z) \oplus_{1}^{n} W^{2 n}(D, \mathbf{H})}
$$

endowed with the Hilbert space norm. Let $M_{z}$ be the multiplication operator with $z$ on $W^{2 n}(D, \mathbf{H})$. Then $\bigoplus_{1}^{n} M_{z}$ is a $C^{2 n}$-scalar subnormal operator and its spectral distribution is

$$
\Phi: \bigoplus_{1}^{n} C_{0}^{2 n}(\mathbf{C}) \rightarrow \mathscr{L}\left(\bigoplus_{1}^{n} W^{2 n}(D, \mathbf{H})\right), \quad \boldsymbol{\Phi}\left(\bigoplus_{1}^{n} f_{i}\right)=\bigoplus_{1}^{n} M_{f_{i}} .
$$

Since $M_{z}$ commutes with $M_{T-z}, \bigoplus_{1}^{n} \widetilde{M}_{z}$ is still a scalar operator of order $2 n$, with $\widetilde{\Phi}$ as spectral distribution.

Let $\bigoplus_{1}^{n} V$ be the operator

$$
(\bigoplus V)\left(\bigoplus h_{i}\right)=\left(1 \otimes h_{1}, \ldots, 1 \otimes h_{n}\right)^{t}+\overline{(T-z) \oplus_{1}^{n} W^{2 n}(D, \mathbf{H})},
$$

from $\bigoplus_{1}^{n} \mathbf{H}$ into $H(D)$, denoting by $\left(1 \otimes h_{1}, \ldots, 1 \otimes h_{n}\right)^{t}$ the constant function $\bigoplus_{1}^{n} h_{i}$. Then

$$
\left(\bigoplus_{1}^{n} V\right) T=\left(\bigoplus_{1}^{n} \widetilde{M}_{z}\right)\left(\bigoplus_{1}^{n} V\right)
$$

In particular, $\operatorname{ran}\left(\bigoplus_{1}^{n} V\right)$ is an invariant subspace for $\bigoplus_{1}^{n} \widetilde{M}_{z}$. In order to conclude the proof of this theorem, it is enough to show Lemma 4.6.

4.6 Lemma. Let $D$ be a bounded disk which contains $\sigma(T)$. Then the operator $\bigoplus_{1}^{n} V: \bigoplus_{1}^{n} \mathbf{H} \rightarrow H(D)$ is one-to-one and has closed range. 
Proof of Lemma 4.6. Let $\bigoplus_{1}^{n} h_{i}^{k} \in \bigoplus_{1}^{n} \mathbf{H}$ and $\bigoplus_{1}^{n} f_{i}^{k} \in \bigoplus_{1}^{n} W^{2 n}(D, \mathbf{H})$ be sequences (in $k$ ) such that

$$
\lim _{k \rightarrow \infty}\left\|(T-z) \bigoplus_{1}^{n} f_{i}^{k}+\bigoplus_{1}^{n}\left(1 \otimes h_{i}^{k}\right)\right\|_{\Theta_{1}^{n} W^{2 n}}=0
$$

It suffices to show that $\lim _{k \rightarrow \infty} \bigoplus_{1}^{n} h_{i}^{k}=0$. The limit given directly above can be written as

$$
\left\{\begin{array}{l}
\lim _{k \rightarrow \infty}\left\|\left(T_{11}-z\right) f_{1}^{k}+T_{12} f_{2}^{k}+\cdots+T_{1 n} f_{n}^{k}+1 \otimes h_{1}^{k}\right\|_{W^{2 n}}=0 \\
\quad \vdots \\
\lim _{k \rightarrow \infty}\left\|\left(T_{j, j}-z\right) f_{j}^{k}+T_{j, j+1} f_{j+1}^{k}+\cdots+T_{j, n} f_{n}^{k}+1 \otimes h_{j}^{k}\right\|_{W^{2 n}}=0 \\
\quad \vdots \\
\lim _{k \rightarrow \infty}\left\|\left(T_{n, n}-z\right) f_{n}^{k}+1 \otimes h_{n}^{k}\right\|_{W^{2 n}}=0 .
\end{array}\right.
$$

In order to prove Lemma 4.6 we need the following:

Fact. For $t=1,2,3, \ldots, n$,

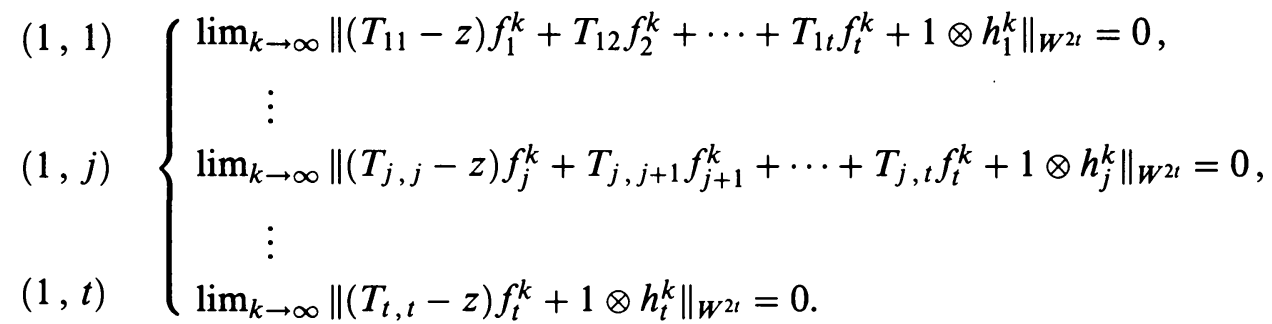

We prove this fact by induction. We assume that Lemma 4.7 holds for some given $t=2,3, \ldots, n$. We only need to verify that

$$
\left\{\begin{array}{l}
\lim _{k \rightarrow \infty}\left\|\left(T_{11}-z\right) f_{1}^{k}+T_{12} f_{2}^{k}+\cdots+T_{1, t-1} f_{t-1}^{k}+1 \otimes h_{1}^{k}\right\|_{W^{2(t-1)}}=0, \\
\quad \vdots \\
\quad \vdots \\
\lim _{k \rightarrow \infty}\left\|\left(T_{t-1, t-1}-z\right) f_{t-1}^{k}+1 \otimes h_{t-1}^{k}\right\|_{W^{2(t-1)}}=0 .
\end{array}\right.
$$

However the reader will note that this result follows directly from $(1,1), \ldots$, $(1, t)$ provided $\lim _{k \rightarrow \infty}\left\|\bar{\partial}^{i} f_{t}^{k}\right\|_{2, D}=0$ for $i=0,1, \ldots, 2(t-1)$. So this will be shown to be true.

Claim 1. $\lim _{k \rightarrow \infty} h_{t}^{k}=0$.

The proof of Lemma 3.5 suitably modified to include the higher order partials with $T=T_{t, t}$ shows the claim to be true.

Claim 2. $\lim _{k \rightarrow \infty}\left\|(I-P) \bar{\partial}^{i} f_{t}^{k}\right\|_{2, D}=0$ for $i=0, \ldots, 2(t-1)$.

By Claim 1 and the equation $(1, t), \lim _{k \rightarrow \infty}\left\|\left(T_{t, t}-z\right) f_{t}^{k}\right\|_{W^{2 t}}=0$. Then 
we can apply Proposition 3.2 and Corollary 3.3 with $T=T_{t, t}$. In fact,

$$
\begin{aligned}
\|(I- & P)\left(f_{t}^{k}, \bar{\partial} f_{t}^{k}, \ldots, \bar{\partial}^{2 t-2} f_{t}^{k}\right) \|_{2, D} \\
\leq & C_{D}\left(\left\|\left(T_{t, t}-z\right)^{*}\left(\bar{\partial} f_{t}^{k}, \ldots, \bar{\partial}^{2 t-1} f_{t}^{k}\right)\right\|_{2, D}\right. \\
& +\|\left(T_{t, t}-z\right)^{*}\left(\bar{\partial}^{2} f_{t}^{k}, \ldots, \bar{\partial}^{2 t} f_{t}^{k} \|_{2, D}\right) \\
\leq & C_{D}\left(\left\|\left(T_{t, t}-z\right)\left(\bar{\partial} f_{t}^{k}, \ldots, \bar{\partial}^{2 t-1} f_{t}^{k}\right)\right\|_{2, D}\right. \\
& \left.+\left\|\left(T_{t, t}-z\right)\left(\bar{\partial}^{2} f_{t}^{k}, \ldots, \bar{\partial}^{2 t} f_{t}^{k}\right)\right\|_{2, D}\right),
\end{aligned}
$$

where $P$ denotes the orthogonal projection of $\bigoplus_{2 t-1} L^{2}(D, \mathbf{H})$ onto the Bergman space $\bigoplus_{2 t-1} A^{2}(D, \mathbf{H})$. Thus Claim 2 follows from $(1, t)$.

By Claim 2, for $i=0,1, \ldots, 2(t-1)$

$$
\lim _{k \rightarrow \infty}\left\|\left(T_{t, t}-z\right) \bar{\partial}^{i} f_{t}^{k}-\left(T_{t, t}-z\right) P \bar{\partial}^{i} f_{t}^{k}\right\|_{2, D}=0 .
$$

Since $\lim _{k \rightarrow \infty}\left\|\left(T_{t, t}-z\right) f_{t}^{k}\right\|_{W^{2 t}}=0$ by Claim 1 and equation $(1, t)$,

$$
\lim _{k \rightarrow \infty}\left\|\left(T_{t, t}-z\right) P \bar{\partial}^{i} f_{t}^{k}\right\|_{2, D}=0
$$

for $i=0,1, \ldots, 2(t-1)$. Since every hyponormal operator has property $(\beta)$ by Lemma 2.1 , for $i=0,1, \ldots, 2(t-1), P \bar{\partial}^{i} f_{t}^{k} \rightarrow 0$ uniformly on compact subsets of $D$.

Consider $\sigma(T) \subset B(0, r) \subset \overline{B(0, r)} \subset D$. For $i=0,1, \ldots, 2(t-1)$,

$$
\begin{aligned}
\left\|P \bar{\partial}^{i} f_{t}^{k}\right\|_{2, D}^{2} & =\int_{D}\left\|P \bar{\partial}^{i} f_{t}^{k}(z)\right\|^{2} d \mu(z) \\
& =\int_{\overline{B(0, r)}}\left\|P \bar{\partial}^{i} f_{t}^{k}(z)\right\|^{2} d \mu(z)=\int_{D \backslash \overline{B(0, r)}}\left\|P \bar{\partial}^{i} f_{t}^{k}(z)\right\|^{2} d \mu(z) .
\end{aligned}
$$

By property $(\beta)$, the first integral converges to 0 . Since $T-z$ is invertible on $D \backslash \overline{B(0, r)}$, the second integral also converges to 0 . Therefore,

$$
\lim _{k \rightarrow \infty}\left\|P \bar{\partial}^{i} f_{t}^{k}\right\|_{2, D}=0 .
$$

From Claim 2, we get $\lim _{k \rightarrow \infty}\left\|\bar{\partial}^{i} f_{t}^{k}\right\|_{2, D}=0$ for $i=0,1, \ldots, 2(t-1)$. So this completes the proof of the fact stated above.

Let us come back now to the proof of Lemma 4.6. By the fact, we get the equation

$$
\lim _{k \rightarrow \infty}\left\|\left(T_{11}-z\right) f_{1}^{k}+1 \otimes h_{1}^{k}\right\|_{W^{2}}=0 \text {. }
$$

By the application of Lemma 3.5 with $T=T_{11}$, we get $\lim _{k \rightarrow \infty} h_{1}^{k}=0$. Since $\lim _{k \rightarrow \infty} h_{j}^{k}=0$ for $j=1, \ldots, n, \lim _{k \rightarrow \infty} h^{k}=0$ where $h^{k}=\left(h_{1}^{k}, \ldots, h_{n}^{k}\right)$. Thus $\bigoplus_{1}^{n} V$ is one-to-one and has closed range.

This also concludes the proof of Theorem 4.5, because $\operatorname{ran}\left(\bigoplus_{1}^{n} V\right)$ is a closed invariant subspace for the scalar operator $\left(\bigoplus_{1}^{n} \widetilde{M}_{z}\right)$.

4.8 Corollary. If $T$ is an algebraic operator, then $T$ is a subscalar operator. Proof. It is clear from Theorem 4.4 and Theorem 4.5. 
4.9 Corollary. Every operator on a finite-dimensional complex space is subscalar.

4.10 Definition. An operator $T \in \mathscr{L}(\mathbf{H})$ is said to have property $(\alpha)$ if for every (not necessarily strict) contraction $A$, every operator $X$ with dense range such that $X A=T X$, and every $h$ in $\mathbf{H}$, there exists a non-zero polynomial $p$ such that $p(T) h \in \operatorname{ran} X$.

4.11 Lemma ([ABFP], Proposition 3.9). If a strict contraction $T$ (i.e., $\|T\|<$ 1) has the property $(\alpha)$, then $T$ is an algebraic operator.

4.12 Corollary. If a strict contraction $T$ has property $(\alpha)$, then $T$ is subscalar.

4.13 Corollary. If $A \prec T$ and $T$ is algebraic, then $A$ is subscalar.

Proof. By hypothesis, we can show $A$ is algebraic.

Remark. Let

$$
B=\left(\begin{array}{ll}
0 & T \\
0 & 0
\end{array}\right)
$$

where $T$ is hyponormal. Then $B$ is a non-hyponormal, but $B$ is a subscalar operator of order 4.

4.14 Definition. An operator $T$ in $\mathscr{L}\left(\bigoplus_{1}^{n} \mathbf{H}\right)$ is said to be a triangular $n$ hyponormal operator if

$$
T=\left(\begin{array}{ccccc}
T_{11} & T_{12} & \ldots & \ldots & T_{1 n} \\
0 & T_{22} & \ldots & \ldots & \\
\ldots & \ldots & \ldots & \ldots & \\
\ldots & \ldots & \ldots & T_{n-1, n-1} & T_{n-1, n} \\
\ldots & \ldots & \ldots & 0 & T_{n n}
\end{array}\right)
$$

where $\left(T_{i j}\right)$ are commuting hyponormal operators on $\mathbf{H}$.

4.15 Corollary. Let $T$ be a triangular n-hyponormal operator. Then $T$ is a subscalar operator.

\subsection{Question. Let}

$$
T=\left(\begin{array}{ll}
T_{1} & T_{2} \\
T_{3} & T_{4}
\end{array}\right)
$$

where $\left\{T_{i}\right\}$ are commuting hyponormal operators. Is $T$ subscalar?

\section{ACKNOWLEDGMENT}

The author wishes to thank Professor Scott W. Brown for his considerable guidance.

\section{REFERENCES}

[ABFP] C. Apostol, H. Bercovici, C. Foias, and C. Pearcy, Quasiaffine transforms of operators, Michigan Math. J. 29 (1982), 243-255.

[Co 1] J. B. Conway, A course in functional analysis, Springer-Verlag, New York, 1985.

[Co 2] __ Subnormal operators, Pitman, London, 1981.

[Ha] P. R. Halmos, Capacity in Banach algebras, Indiana Univ. Math. J. 20 (1971), 855-863.

[Ko] Eungil Ko, Subscalar and quasisubscalar operators, Ph.D. thesis, Indiana University, 1993. 
[MP] M. Martin and M. Putinar, Lectures on hyponormal operators, Birkhauser, Basel and Boston, 1989.

[Pu] M. Putinar, Hyponormal operators are subscalar, J. Operator Theory 12 (1984), 385-395.

Global Analysis Research Center, Department of Mathematics, Seoul National UniVERSITY, SEOUL 151-742, KorEA 